\title{
Gellan gum-based Hydrogel Bilayered Scaffolds for Osteochondral Tissue Engineering
}

\author{
Diana R. Pereira ${ }^{1,2, a}$, Raphael F. Canadas ${ }^{1,2, b}$, Joana Silva-Correia $a^{1,2, c}$, \\ Alexandra P. Marques ${ }^{1,2, d}$, Rui L. Reis ${ }^{1,2, e}$ and Joaquim M. Oliveira ${ }^{1,2, f^{\prime}}$

\begin{abstract}
13B's Research Group - Biomaterials, Biodegradables and Biomimetics, University of Minho, Headquarters of the European Institute of Excellence on Tissue Engineering and Regenerative Medicine, AvePark, S. Cláudio de Barco, 4806-909 Taipas, Guimarães, Portugal

${ }^{2}$ ICVS/3B's - PT Government Associate Laboratory, Braga/Guimarães, Portugal. dapmarques@dep.uminho.pt, ${ }^{\mathrm{e}}$ rgreis@dep.uminho.pt, fmiguel.oliveira@dep.uminho.pt (corresponding author)
\end{abstract} \\ adiana.pereira@dep.uminho.pt, ${ }^{b}$ raphael.canadas@dep.uminho.pt, ${ }^{c}$ joana.correia@dep.uminho.pt,
}

Keywords: Bilayered scaffolds; Gellan gum; Hydrogels; Osteochondral; Tissue engineering.

\begin{abstract}
It has been shown that hydrogel bilayered scaffolds combining cartilage- and bone-like layers are most advantageous for treating osteochondral defects. In this study, it is proposed the use of low acyl gellan gum (LAGG) for developing bilayered hydrogel scaffolds for osteochondral tissue engineering. The cartilage-like layer of the GG-based bilayered hydrogel scaffolds is composed of LAGG (2 wt \%). By adding a $2 \mathrm{wt} \%$ LAGG aqueous solution to different amounts of HAp (5-20 wt\%) it was possible to produce the bone-like layer. In vitro bioactivity tests were performed by means of soaking the LAGG/LAGG-HAp hydrogel scaffolds in a simulated body fluid solution up to 14 days. Scanning electron microscopy, Fourier transform infra-red spectroscopy and X-ray diffraction analyses demonstrated that apatite formation is limited to the bone-like layer of the LAGG/LAGG-HAp bilayered hydrogel scaffolds.
\end{abstract}

\section{Introduction}

Osteochondral defects (OCD) occuring after trauma, cancer, or metabolic disorders is still a major clinical challenge in orthopedics for regeneration and repair. Therefore, for treating an OCD, it should be considered the need for simultaneous regeneration of cartilage and subchondral bone in the same scaffold [1].

Bilayered scaffolds presenting cartilage- and bone-like layers have been proposed by our group for treating OCD [2]. These systems combine biomimetic biphasic scaffolds composed by hydroxyapatite (HAp) and chitosan.

More recently, hydrogels have been attracting great deal of attention for treating OCD. Gellan gum (GG) is a polysaccharide that can form ionic-crosslinked hydrogels with thermosensitive behaviour suitable as injectable formulations that jellify in situ at a temperature close to body temperature. Previously, our group [3-6] demonstrated that GG hydrogels adequately support the growth and ECM deposition of human articular chondrocytes in vitro and in vivo. In another study [6], we modified Gellan Gum by metacrylation, allowing the use of photo-polymerization as an alternative method for hydrogel formation with increased structural and mechanical features. Because of its high water content, GG-based hydrogels are a very promising structure for finding applications in cartilage tissue regeneration.

In this study, low acyl gellan gum (LAGG) was used to produce bilayered hydrogel scaffolds for osteochondral tissue engineering. The bilayered LAGG/LAGG-hydroxyapatite (HAp) hydrogel scaffolds were obtained from $2 \mathrm{wt} \%$ LAGG aqueous solutions (cartilage-like layer), while by adding a $2 \mathrm{wt} \%$ LAGG aqueous solution to different amounts of HAp (5-20 wt\%), it was possible to produce the bone-like layer. The bilayered LAGG/LAGG-HAp hydrogel bioactivity was assessed by 
means of immersion in a simulated body fluid solution up to 14 days. Scanning electron microscopy, Fourier transform infra-red spectroscopy and X-ray diffraction analyses were performed to evaluate apatite formation.

\section{Materials and Methods}

\subsection{Materials}

Low acyl Gellan-gum (LAGG, Gelzan ${ }^{\mathrm{TM}} \mathrm{CM}, \mathrm{F}=1000$ 000) was obtained from Sigma-Aldrich (USA). Unless otherwise stated all the reagents were purchased from Sigma-Aldrich.

\subsection{Gellan gum/Gellan gum-hydroxyapatite hydrogel bilayered scaffolds production}

LAGG was dissolved in distilled water in order to separately prepare two $2 \%(\mathrm{wt} / \mathrm{v})$ batch solutions, For that, the polymer was dissolved in a water-bath at $90^{\circ} \mathrm{C}$, with constant stirring, until completely dissolution. Then, the temperature was decreased until $60^{\circ} \mathrm{C}$ keeping the magnetic stirring. To produce the bone-like part, hydroxyapatite (HAp) was synthesized as described elsewhere [7], and HAp particle of size below $63 \mu \mathrm{m}$ was incorporated in the LAGG. For each formulation, it was added HAp with ratio of 5, 10, 15 and 20\% (wt/v) to a LAGG 2\% (wt/v) solution. Moulds of silicone ( $7 \mathrm{~mm}$ of diameter and $4 \mathrm{~mm}$ of height) were used to prepare the hydrogel bilayered scaffolds. First, $100 \mu \mathrm{L}$ of $2 \%$ LAGG solution containing 5, 10, 15 and 20\% HAp were added into the moulds and then, $100 \mu \mathrm{L}$ of $2 \%$ LAGG solution were placed on top. Finally, the moulds were covered with phosphate buffer solution (PBS) to promote the ionic crosslinking. The bilayered discs were removed from the moulds 30 minutes after PBS immersion and kept in fresh PBS at $4^{\circ} \mathrm{C}$.

\subsection{Simulated Body Fluid [SBF] Test}

The LAGG/LAGG-HAp hydrogels bilayered scaffolds were soaked in a solution of Simulated Body Fluid (SBF) as described by Kokubo et al. [8]. In order to closest resemble the human blood plasma, the SBF solution $\left(\mathrm{Na}^{+} 142.0, \mathrm{~K}^{+}\right.$5.0, $\mathrm{Ca}^{2+} 2.5, \mathrm{Mg}^{2+} 1.5, \mathrm{Cl}^{-} 147.8, \mathrm{HCO}_{3}^{-}$4.2, $\mathrm{HPO}_{4}{ }^{2-}$ 1.0, $\mathrm{SO}_{4}{ }^{2-} 0.5 \mathrm{mM}$ ) was made by dissolving reagent-grade $\mathrm{NaCl}, \mathrm{NaSO}_{4}, \mathrm{NaHCO}_{3}, \mathrm{KCL}, \mathrm{K}_{2} \mathrm{PO}_{4} .3 \mathrm{H}_{2} \mathrm{O}$, $\mathrm{CaCl}_{2}$ and $\mathrm{MgCl}_{2} \cdot 6 \mathrm{H}_{2} \mathrm{O}$ in distilled water. The solution was settled to $\mathrm{pH} 7.4$ and $36.5^{\circ} \mathrm{C}$ by addition of tris-hydroxymethylaminomethane and hydrochloric acid. Each GG/GG-HAp hydrogel bilayered scaffold was immersed in $10 \mathrm{~mL}$ of SBF solution in $50 \mathrm{~mL}$ polystyrene flasks at $36.5^{\circ} \mathrm{C}$ for periods of 1,7 and 14 days. At each time point the hydrogel bilayered scaffolds were removed and intensively rinsed in distilled water. After being frozen at $-80^{\circ} \mathrm{C}$, the hydrogel bilayered scaffolds were freeze-dried for 4 days. All measurements were performed in triplicate.

\subsection{Physicochemical characterization}

Infra-red spectra of powder specimen were recorded on a Perkin-Elmer spectrometer (PerkinElmer 1600 series equipment, USA). Prior analysis transparent KBr (Riedel-de Haen, Germany) pellets of LAGG/LAGG-HAp hydrogel scaffolds (freeze-dried) were prepared by mixing in a ratio of 1:10 sample and $\mathrm{KBr}(\mathrm{wt} / \mathrm{wt})$, followed by uniaxially pressing the powders under vacuum. All spectra were obtained between 4400 and $450 \mathrm{~cm}^{-1}$ at a $2 \mathrm{~cm}^{-1}$ resolution.

X-ray diffractometer (Bruker D8 Discover, Germany) employing $\mathrm{Cu}-\mathrm{Ka}$ radiation was used to investigate the cristallinity and phase content of the hydrogel bilayered scaffolds (freeze-dried) soaked in SBF solution. Data were collected from 6 to 75 ( $2 \theta$ values), with a step size of 0.04 , and a counting time of $2 \mathrm{~s} /$ step.

The hydrogel bilayered scaffolds (freeze-dried) were analyzed to assess surface morphology and pores size by scanning electron microscopy (SEM/EDS, NanoSEM-FEI Nova 200, USA). Prior to the microstructure analysis, specimens were coated with gold using a Fisons Instruments Coater (Quorum/Polaron E6700, UK) with a current set at $18 \mathrm{~mA}$, for a coating time of $120 \mathrm{~s}$. 


\section{Results and Discussion}

Multilayered scaffold systems for orthopaedic applications, namely for osteochondral tissue regeneration have been widely studied $[1,2]$. One of the major concerns while developing these structures relies on a functional and mechanically stable interface between layers. The observation of the hydrogel bilayered scaffolds (Fig. 1) composed of LAGG 2\% (cartilage-like layer) and LAGG $2 \% / \mathrm{HAp}(5 \%, 10 \%, 15 \%$ and $20 \%$ ) (bone-like layer) confirmed the existence of a clear interface between each layer delimiting distinct cartilage-like and bone-like zones. A single integrated bilayered scaffold made by two distinct layers was achieved while keeping total cohesion of the whole structure.

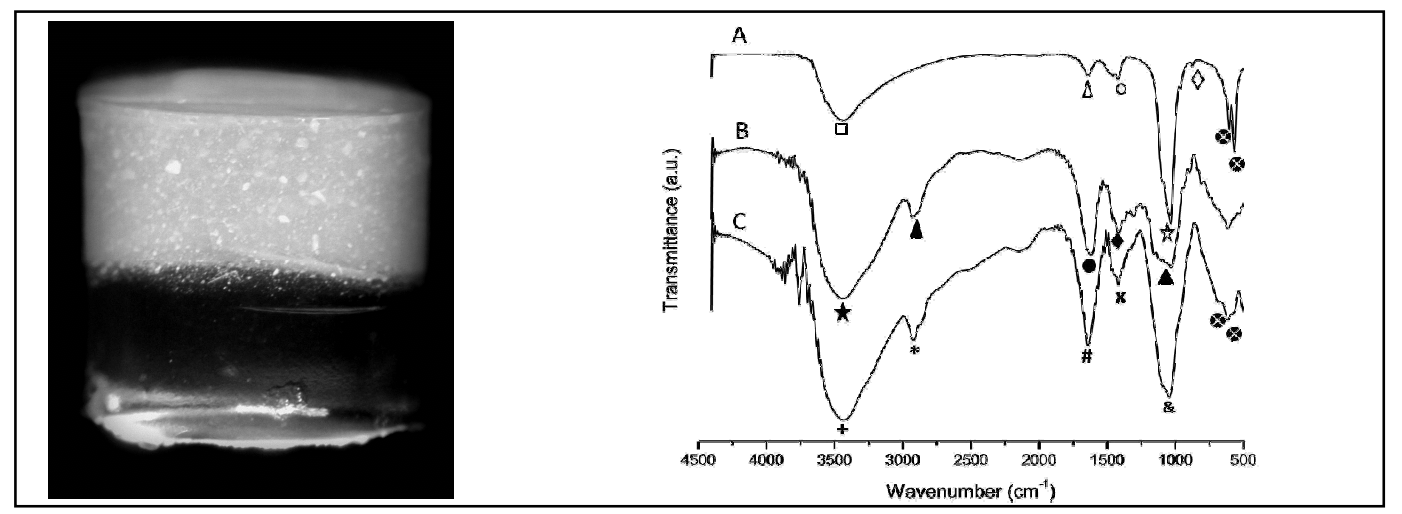

Fig. 1- Macroscopic appearance of the bilayered hydrogels and FTIR spectra of: (A) Hydroxyapatite powder as synthesized; (B) Low acyl Gellan-gum powder, and (C) LAGG /LAGG-HAp20\%.

The physicochemical characterization of the bilayered LAGG-LAGG/HAp scaffolds was performed by Fourier transform infra-red (FTIR) spectroscopy. FTIR was performed in hydrogels bilayered scaffolds and compared with LAGG and HAp powders. Fig. 1 shows the FTIR spectra of HAp, LAGG powders, and LAGG/LAGG-HAp20\% (freeze-dried). The results demonstrated the existence of characteristic bands of HAp, namely at 598 and $559 \mathrm{~cm}^{-1}$, and at $1020 \mathrm{~cm}^{-1}$ attributed to

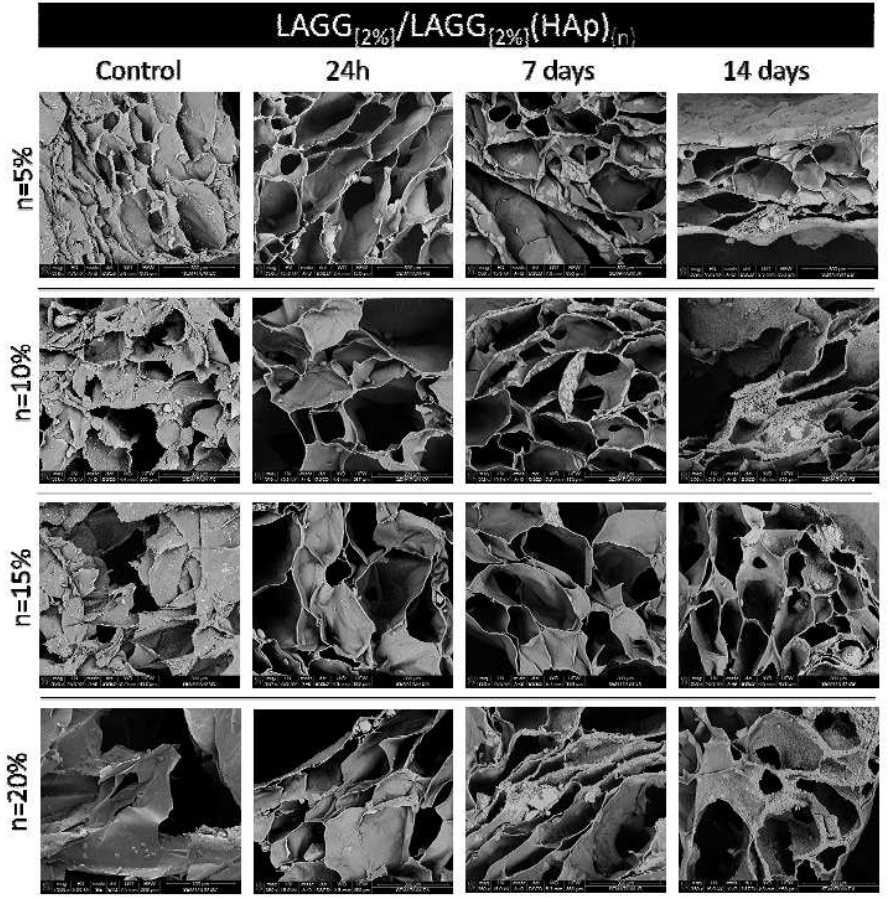

Fig. 2- Cross-sectional SEM photographs of bilayered scaffolds bone-like layer; soaked in SBF solution for 14 days. $\mathrm{P}^{3-}{ }_{4}(v 4)$ and $\mathrm{PO}_{4}^{3-}(v 3)$ groups vibrations, respectively. The typical bands of LAGG were present at $3473 \mathrm{~cm}^{-1}, 2920 \mathrm{~cm}^{-1}, 1618$ $\mathrm{cm}^{-1}, 1536 \mathrm{~cm}^{-1}, 1412 \mathrm{~cm}^{-1}$, and $1037 \mathrm{~cm}^{-1}$ (Fig. 1). New peaks do not appear because the polymer has a molecular composition with the infrared vibrational stretching near the typical peaks of HAp. The peaks resulting from infrared induced stretching are not sufficiently narrow to be possible to see individual peaks. However, an enlargement of the peaks was observed, resulting from the near but different wavenumbers of the vibrational stretching of some molecular bonds present in HAp and LAGG, in the range of 500-600 $\mathrm{cm}^{-1}$, $1000-1200 \mathrm{~cm}^{-1}, 1400 \mathrm{~cm}^{-1}, 1600 \mathrm{~cm}^{-1}$ and $3000-3600 \mathrm{~cm}^{-1}$. This way, the peaks overlaps when the composition of the bilayered scaffold was analyzed.

In presence of functional groups able to trigger apatite nucleation, apatite formation can occur on the surface of 
biomaterials. By using a solution close resembling the ion concentration of human blood plasma [8], in vitro assays were performed in order to verify the bioactivity behavior of the developed bilayered hydrogels. From the control condition in Fig. 2, it is possible to verify that different HAp ratios incorporated within LAGG matrix did not affect the pore size of the scaffolds. The bioactivity test showed that the observed bone-like layer surface has an increasing deposition of precipitates along the incubation time. The formation of calcium phosphates nuclei was noticeably observed after 7 days in SBF. A thicker layer of calcium phosphates was clearly observed after 14 days of incubation. Interestingly, this phenomenon was restricted to the bone-like layer. From Fig. 3, it is also possible to observe the surfaces of the cartilage- and bone-like layers after immersion in SBF solution. The cartilage-like layer did not present any precipitate at the surface, i.e. it shows a completely smooth surface (Fig. 3A). On the contrary, the formation of precipitates, resembling the "cauliflower-like" morphology, was clearly observed in the bone-like layer (Fig. 3B). Though, LAGG did not show apatite-forming ability in SBF. Even containing carboxyl groups, the soaking LAGG hydrogels into SBF did not lead the formation of apatite on its surface, which can be
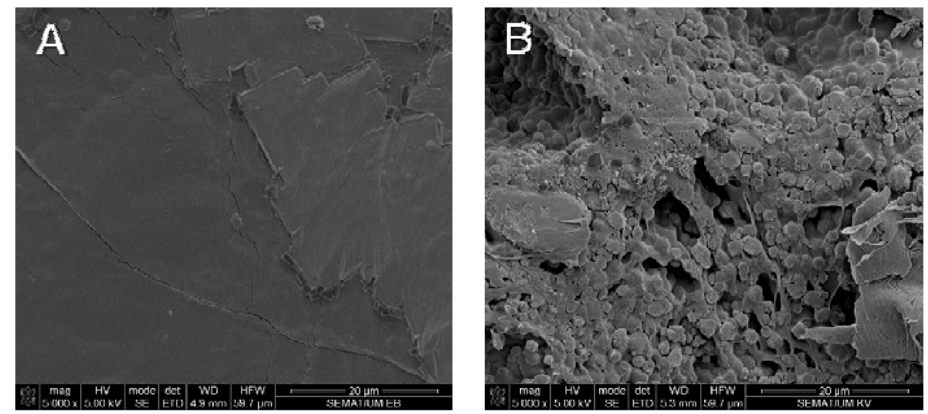

Fig. 3- SEM image of LAGG (cartilage-like layer) (A) and

SEM image of LAGG (20\% HAp; bone-like layer) (B), after 14 days of soaking in SBF.

calcium and phosphate ions from SBF are consumed.

The XRD patterns of the different hydrogel bilayered scaffolds were analyzed after immersion in SBF for 14 days. As the production of the HAp incorporated within LAGG did not comprise sintering, its structure was amorphous. The XRD patterns shown in Fig. 4 for all formulations are the sum of the diffraction pattern of each phase (LAGG, HAp and apatite).

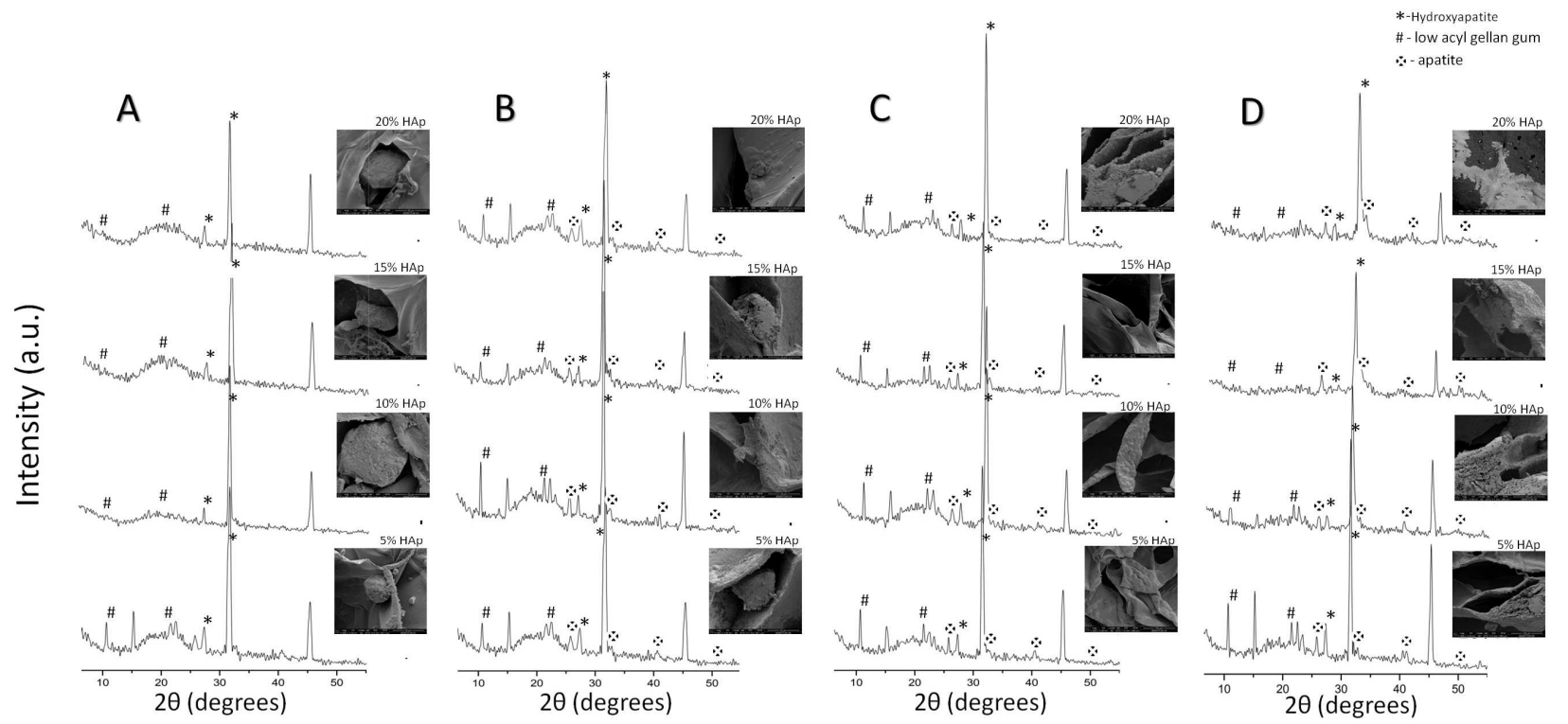

Fig. 4- XRD pattern of bilayered scaffolds soaked in SBF: Control (A), 1 day (B), 7 days (C), and 14 days (D) for the several formulations of LAGG /LAGG (HAp).n; $n=5,10,15$ and $20 \%$.

The intensity of the peaks is directly correlated with the number of crystallites difracting the Xrays. Therefore, HAp finely gorunded presented higher but narrow peaks. In the case of LAGG, a 
typical amorphous material is identified by the sharp peaks appearing in the diffractogram of XRD analysis. The XRD patterns of the hydrogel bilyared scaffolds after soaking in SBF clearly showed the appearing of new peaks corresponding to apatite (Fig. 4). The XRD pattern of the bilayered scaffolds are in agreement with SEM micrographs showing that the appearance of apatite layers correspond to new peaks assignments (Table 1). The intensity of the apatite peaks increased for longer incubation time in SBF. However, the precipitated apatite remained amorphous.

Table 1- XRD peaks assignment (20) of low acyl gellan-gum (LAGG), hydroxyapatite (HAp), and apatite.

\begin{tabular}{|c|c|c|}
\hline $\begin{array}{c}\text { LAGG } \\
(2 \theta \text { assignement })\end{array}$ & $\begin{array}{c}\text { HAp } \\
(2 \theta \text { assignement })\end{array}$ & $\begin{array}{c}\text { Apatite } \\
(2 \theta \text { assignement })\end{array}$ \\
\hline $8.9^{\circ}$ & $31.8^{\circ}$ & $33^{\circ}$ \\
\hline $20.2^{\circ}$ & $25.9^{\circ}$ & $39.8^{\circ}$ \\
\hline & & $46.71^{\circ}$ \\
\cline { 3 - 3 } & - & $50^{\circ}$ \\
\hline
\end{tabular}

\section{Conclusions}

The GG-based bilayered hydrogel scaffolds were successfully produced showing distinct cartilage-like and bone-like layers with an interface that sustains the integrity of the whole structure. The bioactivity test revealed the different behavior of both layer, while in the bone-like layer, a bioactive structure, an apatite-like layer was formed in contact with SBF, the cartilage-like layer did not show a bioactive nature. Therefore, this study demonstrated that the presence of HAp induces apatite nucleation and the released calcium ions accelerate apatite nucleation in the bone-like layer. The gellan gum/gellan gum-hydroxyapatite bilayered hydrogels have a great potential to be used as osteochondral tissue engineering scaffolding.

\section{Acknowledgements}

This study received funding from Portuguese Foundation for Science and Technology (FCT) through the projects OsteoCart (PTDC/CTM-BPC/115977/2009). The authors also thanks FCT for the $\mathrm{PhD}$ scholarship provided to D.R. Pereira (SFRH/BD/81356/2011). The FCT distinction attributed to J.M. Oliveira under the Investigador FCT program (IF/00423/2012) is also greatly acknowledge.

\section{References}

[1] P.B. Malafaya, R.L. Reis. Bilayered chitosan-based scaffolds for osteochondral tissue engineering: Influence of hydroxyapatite on in vitro cytotoxicity and dynamic bioactivity studies in a specific double-chamber bioreactor, Acta. Biomater. 5(2009) 644-60.

[2] J.M. Oliveira, M.T. Rodrigues, S.S. Silva, P.B. Malafaya, M.E. Gomes, C.A. Viegas, et al. Novel hydroxyapatite/chitosan bilayered scaffold for osteochondral tissue-engineering applications: Scaffold design and its performance when seeded with goat bone marrow stromal cells, Biomaterials. 27(2006) 6123-37.

[3] J.T. Oliveira, L.S. Gardel, T. Rada, L. Martins, M.E. Gomes, R.L. Reis. Injectable gellan gum hydrogels with autologous cells for the treatment of rabbit articular cartilage defects, J. Orthop. Res. 28(2010) 1193-9.

[4] J.T. Oliveira, T.C. Santos, L. Martins, R. Picciochi, A.P. Marques, A.G. Castro, et al. Gellan gum injectable hydrogels for cartilage tissue engineering applications: In vitro studies and preliminary in vivo evaluation, Tissue Eng. Part A. 16(2010) 343-53. 
[5] J.T. Oliveira, T.C. Santos, L. Martins, M.A. Silva, A.P. Marques, A.G. Castro, et al. Performance of new gellan gum hydrogels combined with human articular chondrocytes for cartilage regeneration when subcutaneously implanted in nude mice, J. Tissue. Eng. Regen. Med. 3(2009) 493-500.

[6] J. Silva-Correia, J.M. Oliveira, S.G. Caridade, J.T. Oliveira, R.A. Sousa, J.F. Mano, et al. Gellan gum-based hydrogels for intervertebral disc tissue-engineering applications, J. Tissue. Eng. Regen. Med. 5(2011) e97-107.

[7] J.M. Oliveira, S.S. Silva, P.B. Malafaya, M.T. Rodrigues, N. Kotobuki, M. Hirose, et al. Macroporous hydroxyapatite scaffolds for bone tissue engineering applications: Physicochemical characterization and assessment of rat bone marrow stromal cell viability, J. Biomed. Mater. Res. Part A. 91(2009) 175-86.

[8] T. Kokubo, H. Kushitani, S. Sakka, T. Kitsugi, T. Yamamuro. Solutions able to reproduce in vivo surface-structure changes in bioactive glass-ceramic A-W, J. Biomed. Mater. Res. 24(1990) 721-34. 